Article

\title{
Communication Challenges in On-Body and Body-to-Body Wearable Wireless Networks-A Connectivity Perspective
}

\author{
Dhafer Ben Arbia ${ }^{1, *}$, Muhammad Mahtab Alam ${ }^{2}$, Yannick Le Moullec ${ }^{2}$ and Elyes Ben Hamida ${ }^{3}$ \\ 1 SERCOM Lab, Polytechnic School of Tunisia, University of Carthage, B.P. 743, 2078 La Marsa, Tunisia \\ 2 Thomas Johann Seebeck Department of Electronics, Tallinn University of Technology, Ehitajate tee 5, \\ 19086 Tallinn, Estonia; muhammad.alam@ttu.ee (M.M.A.); yannick.lemoullec@ttu.ee (Y.L.M.) \\ 3 IRT SystemX, Building N3, 8 Avenue de la Vauve, CS 90070, 91127 Palaiseau, France; \\ elyes.ben-hamida@irt-systemx.fr \\ * Correspondence: dhafera@qmic.com; Tel.: +974-5010-8593
}

Received: 15 May 2017; Accepted: 30 June 2017; Published: 6 July 2017

\begin{abstract}
Wearable wireless networks (WWNs) offer innovative ways to connect humans and/or objects anywhere, anytime, within an infinite variety of applications. WWNs include three levels of communications: on-body, body-to-body and off-body communication. Successful communication in on-body and body-to-body networks is often challenging due to ultra-low power consumption, processing and storage capabilities, which have a significant impact on the achievable throughput and packet reception ratio as well as latency. Consequently, all these factors make it difficult to opt for an appropriate technology to optimize communication performance, which predominantly depends on the given application. In particular, this work emphasizes the impact of coarse-grain factors (such as dynamic and diverse mobility, radio-link and signal propagation, interference management, data dissemination schemes, and routing approaches) directly affecting the communication performance in WWNs. Experiments have been performed on a real testbed to investigate the connectivity behavior on two wireless communication levels: on-body and body-to-body. It is concluded that by considering the impact of above-mentioned factors, the general perception of using specific technologies may not be correct. Indeed, for on-body communication, by using the IEEE 802.15.6 standard (which is specifically designed for on-body communication), it is observed that while operating at low transmission power under realistic conditions, the connectivity can be significantly low, thus, the transmission power has to be tuned carefully. Similarly, for body-to-body communication in an indoor environment, WiFi IEEE 802.11n also has a high threshold of end-to-end disconnections beyond two hops (approximatively $25 \mathrm{~m}$ ). Therefore, these facts promote the use of novel technologies such as 802.11ac, NarrowBand-IoT (NB-IoT) etc. as possible candidates for body-to-body communications as a part of the Internet of humans concept.
\end{abstract}

Keywords: wearable wireless networks (WWNs); on-body networks (BANs); body-to-body networks (BBNs); connectivity; disaster relief and emergency applications

\section{Introduction}

Internet of humans ( $\mathrm{IoH})$ is a new paradigm in which wearable technology is emerging as a cutting-edge enabler. IoH is the concept of connecting, monitoring and recording human data with the Internet. Wearable technology is revolutionizing many applications, including health-care, sports and fitness, rescue and emergency management, augmented reality, fashion, and so on [1]. Recently, wearable technology revenue has greatly increased, passing from USD 2 billion in 2013 to more than USD 15 billion in 2017. Furthermore, technology ownership has undergone a strong 
increase, from $7 \%$ in 2014 to $14 \%$ by 2015 . By the end of 2017 , it is predicted to double again and reach $28 \%$ [2].

Nowadays people can easily keep track of their health and fitness with human-assistive wearable technology. Elderly people can also be remotely monitored and followed up. For instance, it was recently reported that the UK National Health Service (NHS) could save up to 7 billion pounds per year by using innovative technologies to deliver quality health-care to chronically ill with fewer hospital visits and admissions [1,3].

Virtual and augmented reality (VR/AR) form another showcase of wearable technology which has completely changed the perception of immersive vision. Within next few years, many millions of people will be able to walk around wearing relatively unobtrusive AR devices that offer an immersive and high-resolution view of a visually-augmented world [4]. Among other applications, wearable technology also hails to assist first responders in rescuing and evacuating people during disasters. In past few years, it has been found that wearable technology can be vigorously exploited in disaster contexts to not only save human lives but also to monitor the real-time health status of the rescue team members and victims. Furthermore, it helps operations commanders to make optimal decisions during disaster relief operations.

Other use cases within recent works targeted real testbeds and implementations in order to evaluate the performance of the wearable wireless networks $(\mathrm{WWN})$ integrated with Internet of things (IoT) in real conditions. Miranda et al. in [5] implemented and evaluated a complete common recognition and identification platform (CRIP) for healthcare IoT. CRIP enables a basic configuration and communication standardization of healthcare "things". Other aspects are also covered, in particular security and privacy, and health device integration. Different communication standards were used to deploy CRIP, such as Near Field Communication (NFC), biometrics (fingerprints) and Bluetooth. In most of the above-mentioned applications, wireless communication is inevitable between various types of devices including sensors, actuators, coordinators, and gateways. Additionally, with the advent of body-to-body networks (BBNs or B2B), the communication is extended from classical "on-body networks/body area networks (BANs)" to modern "body-to-body networks (BBNs)" as shown in Figure 1. Consequently, wearable wireless networks (WWNs) are emerging as a new frontier for future smart applications in Internet of things (IoT) and Internet of humans (IoH). From the viewpoint of WWN "connectivity" in IoT and IoH, BBNd provide multi-hop device-to-device (D2D) communication to extend the end-to-end network coverage. This coincides with the vision of 5G, setting up new challenges towards cooperative and collaborative D2D communication among heterogeneous devices.

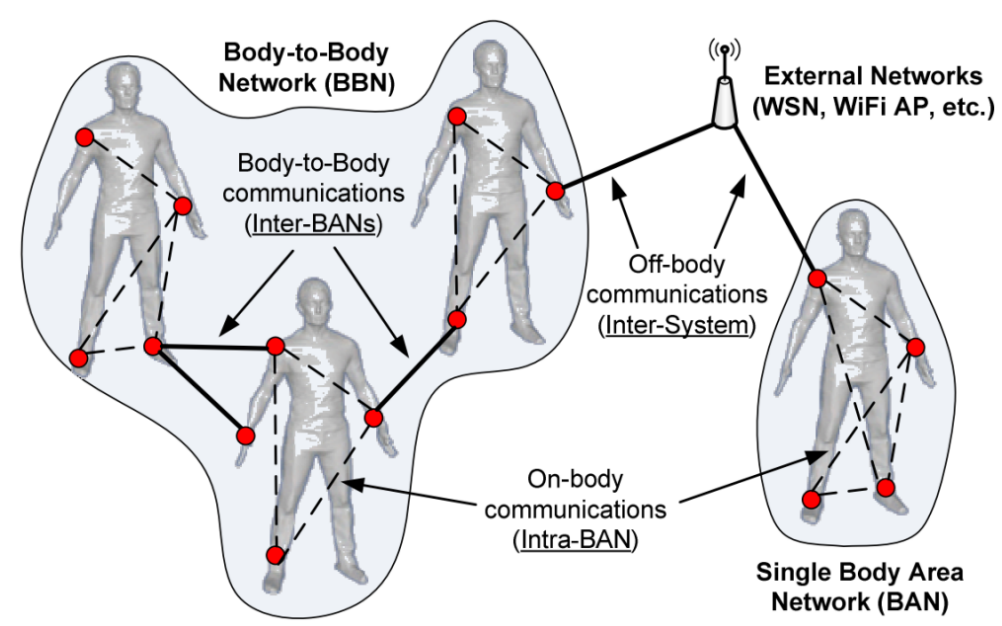

Figure 1. Wearable wireless networks: extending on-body communication to body-to-body and off-body communication. BAN: on-body network. 
The "connectivity" between devices can not be merely ensured by establishing and executing a hand-shaking protocol. For example, if the packet reception ratio is as low as $50 \%$ it means that half of the time devices are disconnected, or in other words, the packets delay recorded is 2 times higher than the given application constraint. Additionally, it also means that only $50 \%$ of the time the considered devices are connected. Consequently, connectivity is an indispensable aspect in WWNs and IoT. To have deeper understanding in this article, we take a closer look at the BAN and BBN communication in WWNs. In particular, we accentuate on the network connectivity challenges in WWNs while considering a first responder rescue and critical operation as a case study.

The contributions of this paper are the following:

- Potential technologies for BAN and BBN wireless networks for the considered application: A comprehensive overview of existing technologies is presented with potential candidates for BAN and BBN communication. We consider various cross-layer design parameters that can have a direct or indirect impact on the connectivity. Most appropriate standards and supported technologies are selected for both BAN and BBN communication in the application context (use case: disaster and relief operations).

- Impact of space-time channel variations, co-channel interference for on-body connectivity: On-body connectivity is addressed from the view points of space-time channel variations and dynamic mobility as well as co-channel interference under the constraints of low power, latency and packet delivery ratio (PDR).

- Real testbed to evaluate and analyze end-to-end connectivity and end-to-end round trip time delay for $B B N$ wireless communication: The analysis of the end-to-end connectivity and the end-to-end round trip time delay behavior for BBN communication is discussed with regards to the data dissemination strategies. Then, the experiment scenario is presented and the testing is detailed based on the newly proposed dedicated routing strategy for a disaster context [6] (Optimized Routing Approach for Critical and Emergency Networks (i.e., ORACE-Net)). This work is a rare implementation of BAN and BBN technologies in the disaster context. It does not only evaluate the considered implementation, but it also provides a strong guidance for possible future emerging candidates for wireless BAN and BBN technologies.

\section{Overview of Candidate Technologies for Wearable Wireless Networks}

While selecting the appropriate technologies for WWNs, there are a number of constraints to be considered. Based on a given application, often power consumption is required to be very low in order to maximize the lifetime of deployed nodes and the whole network. Typically for on-body communication, excessive power can result in additional interference [7] and therefore fine tuning of the transmission power is extremely important. The effective throughput (after adding all overheads) is another constraint directly related with the specific technology being used. In addition, packet latency, dynamic range, node density and network topology are few important constraints.

Below, we summarize possible optimal selection of the technologies for both BAN and BBN communication.

\subsection{Overview of On-Body Communication Technologies and Existing Implementations}

A holistic overview of (non-cellular) existing standards and technologies for WWN applications is presented in Table 1. For on-body networks, over the past decade a legacy has been incurred from the wireless sensor network-powered Zigbee and the IEEE 802.15.4 standard. Such BAN products and protocol designs remained very dominant by covering health-care-related applications such as patient monitoring in hospital wards and intensive care units. Few other variants of this standard (for example IEEE 802.15.4a, mainly used for wireless sensor networks for ultra-wide bands, and IEEE 802.15.4j, a modified physical layer of IEEE 802.15.4) focused on the medical body area network (MBAN) with frequency band between $2360 \mathrm{MHz}$ to $2400 \mathrm{MHz}$ (just before the congested narrow 
band spectrum). However, this is limited to medical-related applications where for instance dynamic mobility, space-time variations of the wireless channel cause less impact and therefore such specific features are not proposed in the standard.

The SmartBAN standard is proposed by the European Telecommunications Standards Institute (ETSI) to support health-care-related applications. However, the proposed BAN-specific features are not scalable for covering other non-medical applications [8]. The IEEE 802.15.6 standard is targeted for wide a range of applications for body area networks. It provides great flexibility, diversity in terms of features and WWN-specific provisions which are necessary to be exploited in the dynamic and emerging applications. The standard proposes BAN-specific channel models (which are very important to accurately model the system performance).

In terms of maximum achievable throughput at narrow-band, the IEEE 802.15.6 standard can reach up to $680 \mathrm{~Kb} / \mathrm{s}$, while operating at maximum frequency and highest modulation order by considering all the overheads of the Media Access Control (MAC) and Physical (PHY) layers [9]. This imposes limits to the use of the IEEE 802.15.6 standard in a few emerging applications. For example in applications such as augmented reality where transmission of high-rate audio and video are necessary, the IEEE 802.15.6 standard does not meet the requirements.

However, since the IEEE 802.15.6 standard provides ultra-low power consumption for both invasive and non-invasive devices while having key security features, it is expected to cover a wide range of applications with relatively low throughput requirements. Importantly, the great flexibility on the usage of multiple options at the PHY (i.e., human body communication, narrow-band, and ultra-wide-band) and at the MAC layer (scheduled access, beacon enabled/disabled, carrier sense multiple access with collision avoidance (CSMA/CA), polling and posting), make the IEEE 802.15.6 standard a viable option for on-body WWN. In this paper we use the IEEE 802.15.6 standard with applications covering the data rate up to $600 \mathrm{kbps}$. Further, we provide an in-depth overview of the standard focused on connectivity. 
Table 1. Comparisons of the key enabling standards for wearable wireless networks [10].

\begin{tabular}{|c|c|c|c|c|c|c|c|}
\hline & $\begin{array}{l}\text { IEEE } \\
802.11 \mathrm{a} / \mathrm{b} / \mathrm{g} / \mathrm{n} \\
(\mathrm{WiFi})\end{array}$ & $\begin{array}{l}\text { IEEE 802.15.1 } \\
\text { (Bluetooth) }\end{array}$ & $\begin{array}{l}\text { IEEE 802.15.1 } \\
\text { (Bluetooth-LE) }\end{array}$ & $\begin{array}{l}\text { IEEE 802.15.4 } \\
\text { (Zigbee) }\end{array}$ & $\begin{array}{l}\text { IEEE 802.15.4a } \\
\text { (UWB) }\end{array}$ & $\begin{array}{l}\text { IEEE 802.15.4j } \\
\text { (MBAN) }\end{array}$ & $\begin{array}{l}\text { IEEE 802.15.6 } \\
\text { (BANs standard) }\end{array}$ \\
\hline $\begin{array}{l}\text { Modes of } \\
\text { Operation }\end{array}$ & $\begin{array}{l}\text { Adhoc, } \\
\text { Infrastructure }\end{array}$ & Adhoc & Adhoc & Adhoc & Adhoc & Adhoc & Adhoc \\
\hline $\begin{array}{l}\text { Physical (PHY) } \\
\text { Layers }\end{array}$ & NB & NB & NB & NB & UWB & NB & NB,UWB,HBC \\
\hline $\begin{array}{l}\text { Radio } \\
\text { Frequencies } \\
\text { (MHz) }\end{array}$ & 2400,5000 & 2400 & 2400 & $868 / 915,2400$ & $\begin{array}{l}75-724 \\
3000-5000 \\
6000-10,000\end{array}$ & $\begin{array}{l}2360-2490 \\
2390-2400\end{array}$ & $\begin{array}{l}402-405,420-450, \\
863-870,902-928, \\
950-956,2360-2400, \\
2400-2438.5\end{array}$ \\
\hline $\begin{array}{l}\text { Power } \\
\text { Consumption }\end{array}$ & $\begin{array}{l}\text { High } \\
(\sim 800 \mathrm{~mW})\end{array}$ & $\begin{array}{l}\text { Medium } \\
(\sim 100 \mathrm{~mW})\end{array}$ & $\begin{array}{l}\text { low } \\
(\sim 10 \mathrm{~mW})\end{array}$ & $\begin{array}{l}\text { Low } \\
(\sim 60 \mathrm{~mW})\end{array}$ & $\begin{array}{l}\text { Low } \\
(\sim 50 \mathrm{~mW})\end{array}$ & $\begin{array}{l}\text { Low } \\
(\sim 50 \mathrm{~mW})\end{array}$ & $\begin{array}{l}\text { Ultra low (1 } \mathrm{mW} \text { at } \\
1 \mathrm{~m} \text { distance })\end{array}$ \\
\hline $\begin{array}{l}\text { Maximal Signal } \\
\text { Rate }\end{array}$ & $\mathrm{Up}$ to $150 \mathrm{Mb} / \mathrm{s}$ & Up to $3 \mathrm{Mb} / \mathrm{s}$ & Up to $1 \mathrm{Mb} / \mathrm{s}$ & Up to $250 \mathrm{~Kb} / \mathrm{s}$ & $\begin{array}{l}\text { Up to } \\
27.24 \mathrm{Mb} / \mathrm{s}\end{array}$ & Up to $250 \mathrm{~Kb} / \mathrm{s}$ & $10 \mathrm{~Kb} / \mathrm{s}$ to $10 \mathrm{Mb} / \mathrm{s}$ \\
\hline $\begin{array}{l}\text { Communication } \\
\text { Range }\end{array}$ & $\begin{array}{l}\text { Up to } 250 \mathrm{~m} \\
(802.11 \mathrm{n})\end{array}$ & $\begin{array}{l}100 \mathrm{~m} \\
\text { (class } 1 \text { device) }\end{array}$ & Up to $100 \mathrm{~m}$ & Up to $75 \mathrm{~m}$ & Up to $30 \mathrm{~m}$ & Up to $75 \mathrm{~m}$ & $\begin{array}{l}\text { Up to } 10 \mathrm{~m} \\
\text { (nominal } \sim 2 \mathrm{~m} \text { ) }\end{array}$ \\
\hline Topology size & $\begin{array}{l}2007 \text { devices for } \\
\text { structured } \\
\text { WiFi BSS }\end{array}$ & $\begin{array}{l}\text { Up to } 8 \text { devices } \\
\text { per Piconet }\end{array}$ & $\begin{array}{l}\text { Up to } 8 \text { devices } \\
\text { per Piconet }\end{array}$ & $\begin{array}{l}\text { Up to } 65,536 \text { devices } \\
\text { per network }\end{array}$ & $\begin{array}{l}\text { Up to } 65,536 \\
\text { devices per } \\
\text { network }\end{array}$ & $\begin{array}{l}\text { Up to } 65,536 \\
\text { devices per } \\
\text { network }\end{array}$ & $\begin{array}{l}\text { Up to } 256 \text { devices } \\
\text { per body, and up to } \\
10 \text { WBANs in } 6 \mathrm{mt}\end{array}$ \\
\hline $\begin{array}{l}\text { Target } \\
\text { Applications }\end{array}$ & Data Networks & Voice Links & $\begin{array}{l}\text { Healthcare, fitness, } \\
\text { beacons, security, etc. }\end{array}$ & $\begin{array}{l}\text { Sensor networks, } \\
\text { home automation, etc. }\end{array}$ & $\begin{array}{l}\text { Short range and } \\
\text { high data rates, } \\
\text { localization, etc. }\end{array}$ & $\begin{array}{l}\text { Short range } \\
\text { Medical Body } \\
\text { Area Networks }\end{array}$ & $\begin{array}{l}\text { Body Centric } \\
\text { application }\end{array}$ \\
\hline $\begin{array}{l}\text { Target BAN } \\
\text { Architectures }\end{array}$ & Off-Body & On-Body & On-Body & $\begin{array}{l}\text { Body-to-Body, } \\
\text { Off-Body }\end{array}$ & Body-to-Body & On-Body & On-Body \\
\hline
\end{tabular}

* NB: Narrow Band, UWB: Ultra-wide-band, HBC: Human body communication. 


\subsection{Body-to-Body Technologies and Implementations}

For BBN communications, the devices are supposed to communicate over relatively long distances and often are equipped with better battery lifetime in comparison to BAN sensors. Table 1 summarizes the BBN communication standards. The distance from one BAN to another is likely to exceed several hundred meters and therefore short-range and low-power technologies (as mentioned for on-body) are not suitable choices. Consequently, the dynamic range constraint eliminates some candidates for BBN communications including IEEE 802.15.6, IEEE 802.15.4 (ZigBee), IEEE 802.15.4j Medical Body Area Network (MBAN) and IEEE 802.15.4a (ultra-wideband, UWB). As can be seen in Table 1, the eligible standards that can fulfill technical and operational requirements for BBN communications are; WiFi IEEE 802.11a/b/g/n and Bluetooth IEEE 802.15.1 (and Bluetooth low energy, BLE). In comparison to Bluetooth, WiFi is the most relevant to BBN communication for the following reasons: (1) the communication range of $\mathrm{WiFi}$, which is up to $250 \mathrm{~m}$ (outdoor), is higher than the range assured by Bluetooth which could reach a maximum of $100 \mathrm{~m}$ (under specific conditions for class 1); (2) there are a large number of mobile devices implementing at least one of the WiFi varieties cited above; and (3) bandwidth assured by WiFi is around $150 \mathrm{Mb} / \mathrm{s}$ (in case of IEEE 802.11n) and could even reach 500 $\mathrm{Mb} / \mathrm{s}$ (IEEE 802.11ac), compared to the hundreds of $\mathrm{Kb} / \mathrm{s}$ offered by Bluetooth.

Furthermore, Bluetooth low energy (BLE) and WiFi are expected to provide short range coverage from 100 to $200 \mathrm{~m}$ with a throughput range from a few Mbps to hundreds of Mbps. BLE or Bluetooth Smart technologies are strong candidates for the BBN communications, however, they remain limited in terms of range (100 $\mathrm{m}$ theoretical) as well as low transmission power and therefore they are high power interference-sensitive. On the other hand, recent variants of the WiFi standard (i.e., IEEE 802.11n, IEEE 802.11ac, etc.) offer provisions to operate in multi-band frequencies (i.e., $2.4 \mathrm{GHz}$ and $5 \mathrm{GHz}$ ). Additionally, using WiFi, devices could operate for more than $10 \mathrm{~h}$ with one battery. Thus, WiFi could be considered as a pertinent candidate for future BBN communications.

Indeed, these above statements were proved through recent extensive simulations in [11], where it was concluded that WiFi IEEE 802.11 has the best performance in the considered application (disaster relief networks) compared to ZigBee and Wireless Body Area Network (WBAN). Furthermore, a recent experiment [12] validated WiFi IEEE 802.11n as a BBN communication protocol for the disaster relief applications despite the limitations detailed in Section 4. To sum up, WiFi IEEE 802.11 standard in general remains the most appropriate technology for BBN communications.

\subsection{Overview of Key WWN Applications and Implementations}

The wireless technologies given above are considered as current and prospective technologies which fulfill the BAN and BBN communications. Wireless technology is selected and implemented depending on the requirements of the applications and use cases. Chen et al. in [13] classify the applications into three main classes: (1) remote health and fitness monitoring; (2) military and training; and (3) intelligent biosensors for vehicle-area-networks. Moreover, authors in [13] discuss a list of research projects and implementations, in particular: Advanced Health and Disaster Aid Network (AID-N) [14] targets disaster and public safety applications. AID-N uses wired connection for BAN communication, mesh and ZigBee for BBN. Off-body communication in AID-N are fulfilled through $\mathrm{WiFi}$, cellular networks and the Internet. AID-N aims to sense pulse, blood pressure, temperature and Electrocardiography (ECG). Negra et al. in [15] focus more on the main medical applications: (1) telemedicine and remote patient monitoring; (2) rehabilitation and therapy; (3) biofeedback; and (4) ambient assisted living. The latter work discusses also the Quality of Service (QoS) requirements for the medical context. The earliest proposed schemes target to enhance the on-body devices transmission reliability and improve energy efficiency. Chen et al. in [16] proposed a novel cross-layer design optimization (CLDO) scheme. Indeed, the design of CLDO relies on the three lower layers (i.e., PHY, MAC and network layer). Power consumption is firstly optimized by selecting optimal power relays. Then, the remaining energy in leaf nodes is utilized to increase the lifetime and the reliability. An optimal packet size is given for energy efficiency. Chen et al. claim that an inevitably slight overhead 
accompanies CLDO processing for different factors. First, during network initialization complex procedures are run. Second, the algorithm uses a certain number of iterations which influences the overall performance. Third, CLDO lacks the capacity to manage dynamic location situations.

Recent optimization of existing approaches has been proposed to draw a mathematical model for joint single path routing and relay deployment in BAN design. An interesting algorithm has been introduced by $\mathrm{D}^{\prime}$ Andreagiovanni et al. in [17] to handle the uncertainty that affects traffic demands in the multiperiod capacitated network design problem (MP-CNDP). Additionally, a hybrid primal heuristic based on the combination of a randomized fixing algorithm was proposed by the authors, inspired from ant colony optimization and exact large neighborhood search. Performance of the proposed model has been confirmed based on computational experiment compared to existing solutions. This strategy has been improved by D'Andreagiovanni et al. in [18]. The authors adopt a best performance solution based on a min-max approach [19]. Indeed, the proposed algorithm relies on a combination of a probabilistic fixing procedure, guided by linear relaxations, and an exact large variable neighborhood search [17]. This combination has been inspired by the solution methods approximate nondeterministic tree-search (ANTS) [20], ant colony optimization [21] and other randomized algorithms [22]. D'Andreagiovanni et al. extended their preliminary work [18] by new integer linear programming (ILP) heuristic to solve the design problem. The new techniques detailed in [23] do not only fix the variables expressing routing decisions, but also employ an initial deterministic fixing phase of the variables modeling the activation of relay nodes. Experiment conducted by this work shows that the proposed approach outperforms the existing optimization solvers strategies and the results recorded in [18]. In [24], a heuristic min-max regret approach has been developed for BAN design, showing a significant reduction in the conservatism of optimal solutions with respect to the pure min-max approach of [18].

The main challenges in WWN are around routing techniques for BAN and BBN networks. We have recently proposed a new routing approach (i.e., ORACE-Net) which is dedicated to disaster and critical emergency networks. ORACE-Net [6] relies on end-to-end link quality estimation for routing decisions. The scope of this work is to present the network connectivity analysis of our proposed approach [6]. Another approach presented by Tsouri et al. in [25] relies on Dijkstra's algorithm augmented with novel link cost function designed to balance energy consumption across the network. This latter technique avoids relaying through nodes which spend more accumulated energy than others. Indeed, routing decisions are made based on the energy optimization. Authors claim that the proposed approach increases the network lifetime by $40 \%$ with a slight raise of the energy consumed per bit. This work is limited because the main concern of an operational application is studying the BBN network connectivity and routing which consists of the only present backbone in case of operational and dynamic context.

\section{On-Body Communication and Connectivity}

Typically, the on-body communication architecture is composed of sensors (to obtain physiological data), actuators (to act on obtained observations) and a coordinator (to control and coordinate both on-body and beyond body networks). Sensors (i.e., biological sensors, environment sensors, location and position sensors, etc.) could be connected directly to the on-body coordinator, which is often considered as more powerful with longer lifetime batteries than deployed sensors. Successful connectivity between on-body nodes and coordinators are often constrained due to ultra-low power consumption, processing and storage, data throughput or packet reception ratio and latency. These fine-grain constraints are often impacted by coarse-grain factors such as dynamic and diverse mobility (e.g., for sports and fitness applications), radio-link and signal propagation (indoor, outdoor, underwater, during emergencies and disasters etc.), interference management (co-channel, inter-channel) and coexistence strategies (time-shared, channel-hopping, collaborative etc.), and data dissemination schemes, as well as routing approaches. Consequently, to successfully support 
diverse wearable applications, the vital impact of coarse-grain factors is extremely important to analyze. In this section such factors are explored and presented.

\subsection{Space-Time Varying Radio-Links and Signal Propagation}

Accurate channel modeling is a very active topic of research in both BAN and BBN. In particular, under dynamic and diverse mobility patterns, based on the positions of on-body sensors, space and time varying radio-links can severely affect the connectivity between sensors-coordinator communication.

Such space-time varying characteristics often provide higher degree of correlation as identified in [26]. Further, both short-term and long-term channel fading can be modeled to precise the path-loss factors from signal propagation. Moreover, body shadowing also has to be taken into account for accurate modeling of on-body and body-to-body links [27].

In the IEEE 802.15.6 standard, the proposed channel models are limited to stationary radio-links, which consequently requires space-time variations and accurate signal propagation enhancements. At the narrow band, both proposed channel models (i.e., CM3-A and CM3-B) are distance-dependent; the path-loss derived for those models from measurement campaigns was recently enhanced using bio-mechanical mobility and deterministic models [28]. As an example shown in Figure 2, for a space-time varying link such as "wrist-chest" the average peak-to-peak path-loss is 10-dBs higher than for IEEE 802.15.6 standard channel models and hence is more accurate. Realistic radio-link conditions and signal propagation are important to analyze the true connectivity between on-body sensors-coordinator communication.

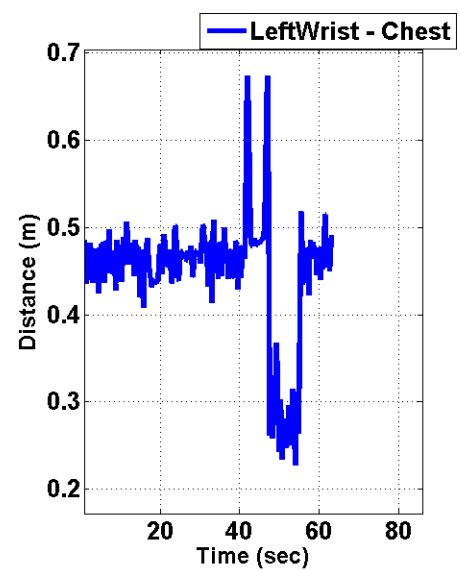

(a)
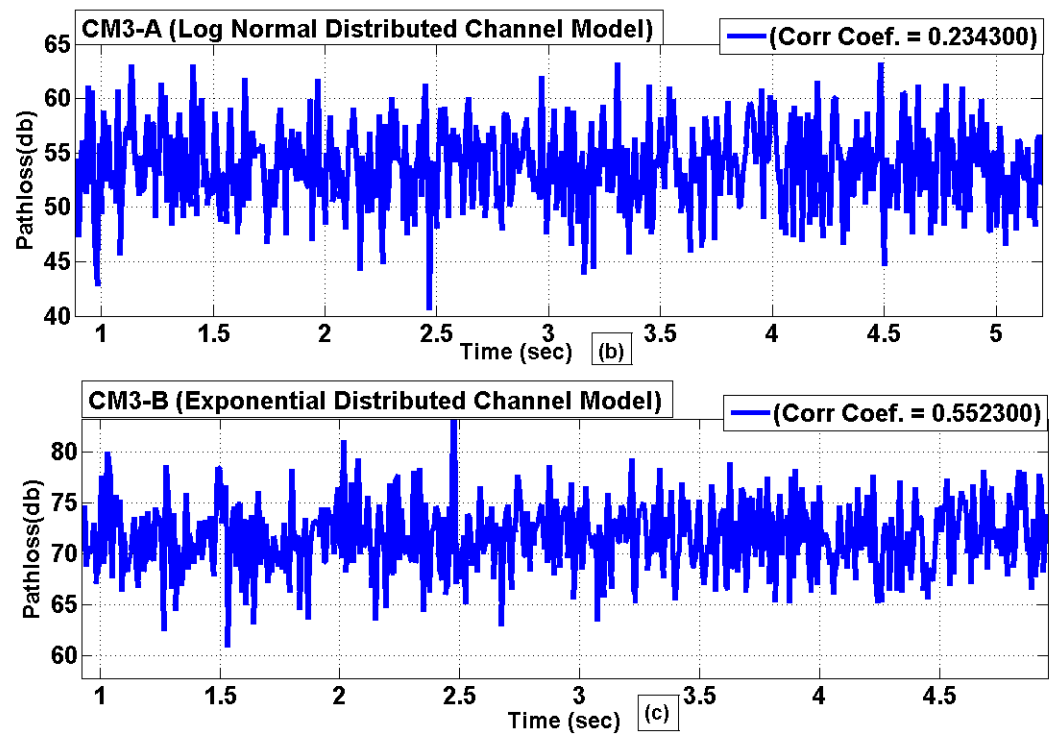

Figure 2. IEEE 802.15.6 enhanced path-loss models obtained from bio-mechanical deterministic channel model. For example, a link between left wrist and chest is shown. (a) Time-varying distances; (b) Enhanced pathloss model CM3-A and (c) enhanced pathloss model CM3-B.

Figure 3 shows the average packet delivery ratio (PDR) results of the on-body communication. Twelve sensors placed on various locations around the body include links which provide space-time variations, static as well as periodic line-of-sight (LOS) and non-line-of-sight (NLOS) links. The results are presented for walking, standing-sitting and running mobility conditions. In addition to transmitting power variations (from $0 \mathrm{dBm}$ to $-20 \mathrm{dBm}$ ), various configurations of the physical layers of the IEEE 802.15.6 standards are exploited. Configuration 1 (C-1) is based on $900 \mathrm{MHz}, 101.2 \mathrm{Kbps}$ and 16 bytes of packet size. Configuration 2 (C-2) and configuration 3 (C-3) differ from C-1 only by the packet sizes which are 128 bytes and 256 bytes, respectively. The last configuration (C-4) is based on 
$2450 \mathrm{MHz}, 971: 4 \mathrm{Kbps}$ and 128 bytes of packet length. It can be seen that, at very low transmission power (such as $-10 \mathrm{dBm}$ and $-20 \mathrm{dBm}$ ), the PDR starts decreasing sharply. Therefore, it is important that, while operating at very low transmission power and under realistic conditions, the packet reception performance can be significantly degraded. Consequently, such power optimizations and fine tunning have to be managed with care to ensure robust connectivity.
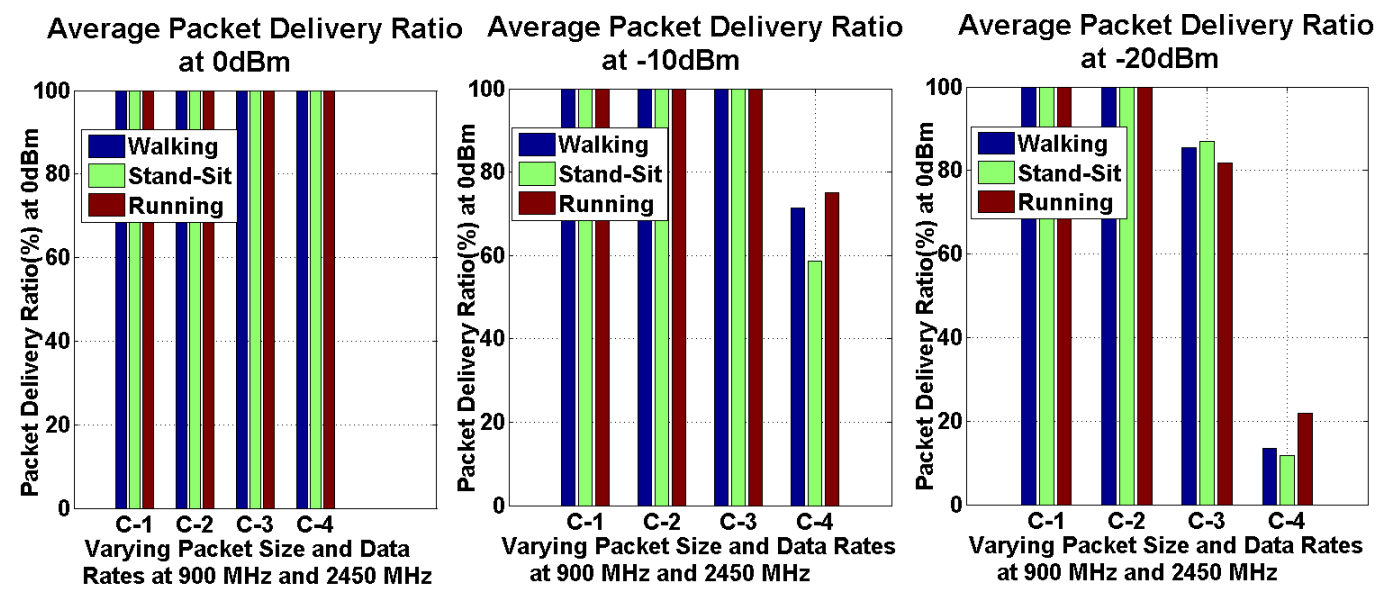

Figure 3. Average packet delivery ratio under walking, stand-sit and running mobility patterns at varied transmission power from $0 \mathrm{dBm},-10 \mathrm{dBm}$ and $-20 \mathrm{dBm}$.

\subsection{Co-Channel Interference and Coexistence Techniques}

With the widespread deployment of wireless networks in our daily living environment, BAN solutions are subject to strong co-channel interference, especially on the unlicensed industrial, scientific and medical (ISM) radio bands which are presently populated by various wireless technologies. The resulting interference can severely impact the connectivity and thus the communication performances.

In this regard, the IEEE 802.15.6 standard has proposed specific coexistence strategies, including beacon shifting, channel hopping, and active super-frame interleaving. In the first approach, each BAN coordinator adopts a different beacon shifting pseudo-random sequence to reduce the interference with neighboring BANs.

The second technique, which is only applicable to narrow-band channels, consists of choosing polynomial-based channel hopping sequences to avoid having neighboring BANs use the same radio channel. The active super-frame interleaving technique enables BAN coordinators to cooperatively coordinate the schedule of their active super-frames. Additionally, the carrier sense multiple access with collision avoidance (CSMA/CA) medium access control protocol could also be adopted to reduce the interference by letting the BANs nodes sense the occupancy of the radio channel prior to any data transmission.

The packet error rate distributions of co-channel interference among up-to five co-located BANs are presented in Figure 4. IEEE 802.15.6 proposed coexistence technique comparisons are highlighted. It is observed that both time shared and channel hopping approaches are well-suited to minimizing interference from neighboring BANs. However, for a dense deployment, new or enhanced schemes must be proposed. 
(a)

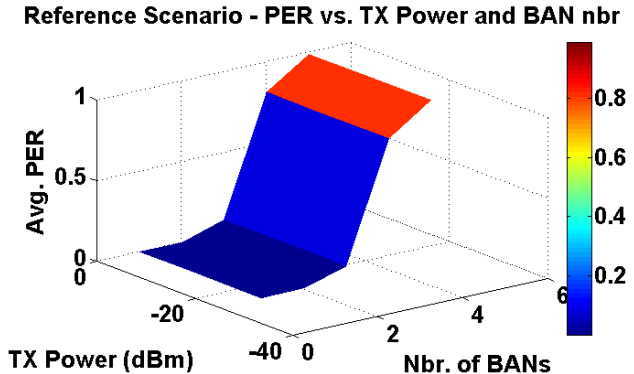

(c)

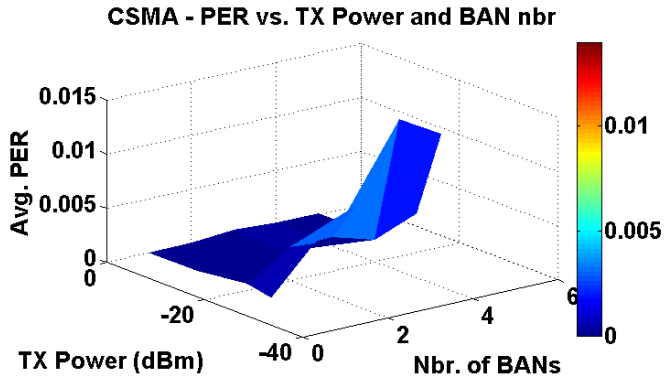

(b) Channel Hopping - PER vs. TX Power and BAN nbr

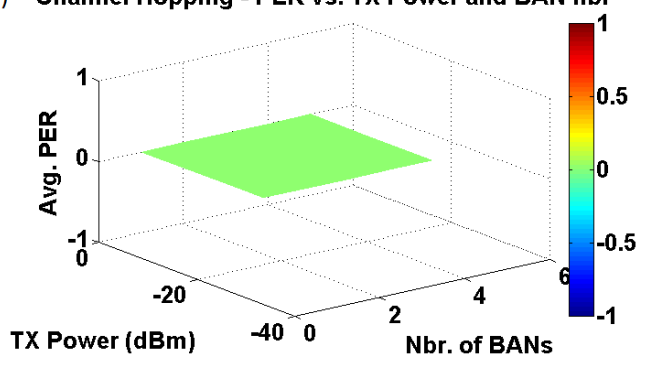

(d) Time Shared - PER vs. TX Power and BAN nbr

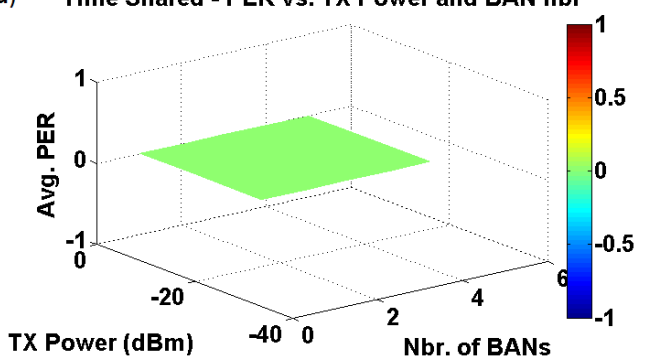

Figure 4. Packet error rate distributions of co-channel interference and evaluation of the IEEE 802.15.6 proposed coexistence techniques. (a) Reference scenario, without any coexistence strategy; (b) Channel hopping; (c) Carrier sense multiple access with collision avoidance (CSMA-CA)-based coexistence;

(d) Time-shared coexistence.

\subsection{Transceiver Implementations and Architecture Considerations}

This section gives an overview of existing IEEE 802.15.6 transceiver (front-end and possible digital baseband part) implementations and highlights the key architectural elements typically found in such implementations. Given the high flexibility of the IEEE 802.15.6 standard, few industrial chips only implement the part of IEEE 802.15.6 standard. As illustrated below, most of the publicly documented implementations are multi-mode, e.g., supporting 802.15.6 and Bluetooth and/or Zigbee.

A few years ago, the authors of $[29,30]$ implemented a $0.13-\mu \mathrm{m}$ Complementary Metal-Oxide-Semiconductor (CMOS) front-end chip supporting the IEEE 802.15.6 NB PHY draft and BT-LE 4.0 standards, as well as proprietary protocols. The chip is composed of a $2.4-\mathrm{GHz}$ sliding-IF receiver, a 2.4-GHz polar loop modulator transmitter, a 900-MHz loop modulator transmitter, and a low frequency 10-bit Successive AppRoximation Digital-Analog Conversion (SAR ADC) for bio-telemetry data acquisition, as well as several peripherals and digital interfaces for an Field-Programmable Gate Array (FPGA)-based digital PHY/MAC design. The chip achieves up to $1000 \mathrm{ksps}$ and requires power between 1.7 and $12.3 \mathrm{~mW}$, depending on the selected mode. It can operate both on the $2.36 \mathrm{GHz}$ MBANs spectrum and the worldwide $2.4 \mathrm{GHz}$ ISM band. It also features a transmitter for operation in China, the EU, North America and Japan (780/868/915/950 MHz, respectively).

The implementation of a $0.18 \mu \mathrm{m}$ CMOS reconfigurable sliding-IF transceiver targeting $400 \mathrm{MHz} / 2.4 \mathrm{GHz}$ IEEE 802.15.6/ZigBee WBAN hubs is presented in [31]. The receiver part comprises a wideband front-end and a reconfigurable amplifier-mixer. The transmitter part comprises a reconfigurable two stage full quadrature mixer, and a delta-sigma fractional-N Phase Locked Loop (PLL), as well as some auxiliary circuits. The chip can operate in the $0.36-0.51 \mathrm{GHz}$ and the 2.36-2.5 $\mathrm{GHz}$ ranges. Its power consumption ranges between $13.2 \mathrm{~mW}$ and $18 \mathrm{~mW}$.

The IEEE-802.15.6-compliant transceiver targeting a multichannel electro-acupuncture application is presented in [32]. As opposed to the two works listed above, this implementation builds upon the Human Body Communication (HBC) physical layer. The chip is implemented on a $0.13-\mu \mathrm{m}$ CMOS process. The possible data rates are $164,328,626 \mathrm{~Kb} / \mathrm{s}$ and $1.3125 \mathrm{Mb} / \mathrm{s}$; its peak power is $5.5 \mathrm{~mW}$ (receiver-activated). The works presented in $[33,34]$ deal with the transceiver and baseband parts, respectively, for a IEEE802.15.6/Bluetooth Low Energy/Zigbee system. The transceiver is implemented 
on a 90-nm CMOS process. The transmitter comprises a 2-point fractional-N PLL-based frequency modulator (FM), and a Delta-Sigma digital-controlled polar Power Amplifier (PA). The receiver builds upon a sliding-IF architecture and operates on the 2.36/2.4 GHz bands. The rates supported by the transceiver are $1 \mathrm{Mbps}$ for BT-LE, $250 \mathrm{kbps}$ for IEEE 802.15.4 (ZigBee), and $971 \mathrm{kbps}$ for IEEE 802.15.6. It also supports a proprietary $2 \mathrm{Mb} / \mathrm{s}$ mode for data-streaming applications such as hearing aids. Its power consumption is $3.8 \mathrm{~mW}$ for the receiver and 4.6-4.4 $\mathrm{mW}$ for the transmitter.

The digital baseband part is implemented on a 40-nm low-power CMOS process. It comprises the transmitter and receiver digital baseband modules, and sub-modules responsible for processing at the PHY and Down Layer (DL) layers. Its power consumption is $200 \mu \mathrm{W}$ for the receiver and $80 \mu \mathrm{W}$ for the transmitter. Its data rates are identical to those of the transceiver described above. More recently, the authors of [35] designed and implemented an IEEE 802.15.6-compliant transceiver building upon the HBC physical layer (as also done in [32]). The analog front-end consists of a transmitter (transmit filter and output electrode) and a receiver (gain stage with automatic gain control and a hard decision detector). The digital part is implemented on a Xilinx Virtex 5 FPGA. The design reaches $763 \mathrm{Kbps}$ (bit error rate of 0.21 ). The dynamic power consumption of the design is $4.5 \mathrm{~nJ} / \mathrm{bit}$ with a spreading factor of 8 (it varies approximately linearly with the spreading factor).

The essential properties of these implementations are summarized in Table 2. As can be seen, implementations have been proposed for either NB or HBC PHY; typically those that support NB PHY also support BT-LE and/or Zigbee The achieved data-rates are either spot-on with the standard, or slightly below or above. However, it can be noted that the IEEE 802.15.6-compliant chipsets are yet to be commercially widely available.

Table 2. Essential properties of IEEE 802.15.6 publicly documented implementations.

\begin{tabular}{|c|c|c|c|c|c|}
\hline $\begin{array}{l}\text { Referred } \\
\text { Works }\end{array}$ & [31] & {$[29,30]$} & {$[33,34]$} & [32] & [35] \\
\hline Standard(s) & $\begin{array}{l}\text { IEEE802.15.6 } \\
\text { NB; Zigbee }\end{array}$ & $\begin{array}{c}\text { IEEE802.15.6 NB; } \\
\text { BT-LE 4.0; } \\
\text { proprietary }\end{array}$ & $\begin{array}{l}\text { IEEE802.15.6 NB; } \\
\text { BT-LE; Zigbee }\end{array}$ & $\begin{array}{l}\text { IEEE802.15.6 } \\
\text { HBC }\end{array}$ & $\begin{array}{l}\text { IEEE802.15.6 } \\
\text { HBC }\end{array}$ \\
\hline Frequency(ies) & $\begin{array}{l}0.36-0.51 \mathrm{GHz} \\
2.36-2.5 \mathrm{GHz}\end{array}$ & $\begin{array}{l}\text { 2.36 GHz; } 2.4 \mathrm{GHz} \\
\text { 780/868/915/950 MHz }\end{array}$ & $2.36 \mathrm{GHz} ; 2.4 \mathrm{GHz}$ & $21 \mathrm{MHz}$ & $21 \mathrm{MHz}$ \\
\hline Data-rates & $\mathrm{N} / \mathrm{A}$ & $1000 \mathrm{kbps}$ & $\begin{array}{c}250 / 971 \mathrm{kbps} \\
2 \mathrm{Mb} / \mathrm{s}\end{array}$ & $\begin{array}{c}164 ; 328 \\
626 \mathrm{~Kb} / \mathrm{s} \\
1.3125 \mathrm{Mb} / \mathrm{s}\end{array}$ & $763 \mathrm{kbps}$ \\
\hline Power & $13.2-18 \mathrm{~mW}$ & $1.7-12.3 \mathrm{~mW}$ & $\begin{array}{c}\text { RX: } 3.8 \mathrm{~mW}+ \\
200 \mu \mathrm{T} ; \mathrm{TX:} \\
4.6-4.4 \mathrm{~mW}+80 \mu \mathrm{W}\end{array}$ & $5.5 \mathrm{~mW}$ (peak) & $4.5 \mathrm{~nJ} / \mathrm{bit}$ \\
\hline Front-end & $0.18 \mu \mathrm{m}$ CMOS & $0.13 \mu \mathrm{m}$ CMOS & $90 \mathrm{~nm}$ CMOS & $0.13 \mu \mathrm{m}$ CMOS & $\begin{array}{c}\text { Discrete } \\
\text { components }\end{array}$ \\
\hline $\begin{array}{c}\text { Digital } \\
\text { baseband }\end{array}$ & $\mathrm{N} / \mathrm{A}$ & $\begin{array}{c}\text { FPGA (not } \\
\text { documented) }\end{array}$ & $40 \mathrm{~nm}$ LP-CMOS & $\mathrm{N} / \mathrm{A}$ & Xilinx Virtex 5 \\
\hline
\end{tabular}

This section focuses on on-body communication. In what follows, we carry on the discussion with the next communication tier, i.e., body-to-body communication.

\section{Body-to-Body Communication and Connectivity}

Due to the growing number of connected devices (smart-phones, computers, game consoles, sensors, and wireless gadgets) to Internet, every human being is considered as a part of a BBN network that could be deployed at any time, anywhere in a context of Internet of humans (IoH) or Internet of things (IoT). Therefore, diverse deployment strategies are possible and various drawbacks are likely to be faced by the BBN network connectivity in a real deployment. Connectivity depends on the following factors: (1) dissemination strategy based on which data is transmitted among the wireless network; (2) communication range: which depends on the used wireless standard (i.e., BAN/MBAN, 
BLE, ZigBee, etc.); (3) routing protocol which must be appropriate for the application context; and (4) experiment area: indoor/outdoor with natural/artificial obstacles. So, let us focus first on the various dissemination and routing techniques with regard to their impact on connectivity while meeting the applications requirements.

\subsection{Impact of Dissemination Strategies on Connectivity}

Data dissemination defines the interconnection logic hierarchy. First, we define the flat wireless network which is a set of nodes communicating with any reachable neighbors using the same wireless technology. Second, a hierarchical wireless network is a set of nodes which communicate with other neighbors from the same hierarchical level only. Specific or elected nodes only could communicate with higher level nodes. The interconnection hierarchy could be set physically (different frequencies to separate nodes into groups, different wireless technologies: more than one WiFi standard, one standard and different channels, etc.) or logically (i.e., same wireless technology and frequencies but different nodes groups related to one specific gateway node). We identify two main dissemination strategies in BBN communications related to the two different interconnection hierarchies.

The clustered data dissemination strategy consists of dividing the network into small groups of sub-networks called clusters. Each cluster leader is called a cluster head $(\mathrm{CH})$. Nodes in a same cluster only communicate with their $\mathrm{CH}$. $\mathrm{CHs}$ communicate between each others to reach wide networks. This strategy fulfills relevant operational requirements for indoor scenarios [36]. Even though this strategy defines a clear hierarchical communication charter, but it still restricts the connection capabilities only through $\mathrm{CH}$. Indeed, when a $\mathrm{CH}$ of a cluster " $\mathrm{A}$ " is out of range from a neighbor cluster " $\mathrm{B}$ ", a connection can not established even if a normal node from " $\mathrm{A}$ " is too close to " $\mathrm{B}$ ". Electing new $\mathrm{CH}$ for the cluster " $\mathrm{A}$ " causes a significant delay, in addition to disconnection during the election process. Therefore, defining members for each cluster is always challenging. Consequently, connectivity of the entire BBN network depends on the connectivity between the CHs.

The distributed data dissemination strategy allows any node in the network to communicate with any reachable node. As an example, an on-body sensor placed on the right wrist could communicate with the on-body coordinator of the neighboring body. This strategy decreases the average delay of the packet compared to the clustered approach [36], whereas, it decreases the link spectral efficiency and the network overall throughput. Accordingly, the average end-to-end connectivity decreases with the link spectral efficiency.

Distributed data dissemination strategies have higher routing overhead than the clustered strategies, since in the first category, any node is allowed to send data to any node. However, in the clustered strategy, the routing overhead remains only between $\mathrm{CHs}$. A delay in route refreshment in the routing table may increase routes unavailability. Therefore, a node with unavailable routes in its routing table is considered as a disconnected node.

\subsection{Impact of Routing Protocols on Connectivity}

In BBN networks, implemented routing protocols refer to various classes. Indeed, recent research tends to evaluate and implement ad hoc routing networks: proactive, reactive, geographic-based and gradient-based [6] in BBN communications. Moreover, context-aware protocols were proposed with regards to the implementation context (i.e, health-care, emergency, operational assistance, military, etc.). Routing protocols consist of the mechanisms that carry data from source to destination which have key role in BBN connectivity. Indeed, routing decisions are based on certain metrics depending on the use case. With regards to the study and experiments conducted by Mekikis et. al in [37], it is claimed that connectivity depends on the networking model (unicast, multicast or broadcast). Ad hoc networks, implemented in the disaster context application, use broadcast for neighbor discovery, and unicast/multicast for routing data. Based on our recent experiment [12], the network performance increases with the accurate end-to-end link quality estimations and real link conditions. 


\subsection{Real Indoor Experiment Scenario: A Wireless Body-to-Body Connectivity Evaluation}

In order to evaluate the connectivity in indoor wireless body-to-body network scenario, we rely on the recent routing approach (i.e., Optimized Routing Approach for Critical and Emergency Networks: ORACE-Net) which is designed specifically for disaster scenario. The scenario consists of a group of people (we are considering two rescuers in this work) moving in/out an office inside a building following a disaster mobility pattern generated by Bonnmotion [38], which is a mobility scenario generation and analysis tool. Each WBAN consists of an android mobile node collecting live data from on-body Shimmer [39] sensors as depicted in Figure 5b. Additionally, four tactical static nodes (numbered from 2 to 5 in Figure 5a) are deployed during the disaster scenario which represent a temporary backbone through which data is routed to Internet. A dedicated node in the network is considered as a gateway, called a command center node (CC) placed in the back gate of the office as shown in Figure 5a. The CC relays data from the deployed network to Internet and vice versa. Our emphasis of the evaluation mainly concerns the connectivity of the WBAN mobile nodes. To that end, the tactical nodes placements are selected as such to enhance the signal propagation and as a result increase the end-to-end connectivity between mobile WBANs and Internet (through the CC node). Experiment scenario map is depicted in Figure 5a. The experimental parameters are given in Table 3.

Table 3. Experimental parameters and configuration settings. ORACE: Optimized Routing Approach for Critical and Emergency Networks; CC: command center node.

\begin{tabular}{ll}
\hline \multicolumn{1}{c}{ Parameter } & General Settings \\
\hline Number of WBANs & \multicolumn{1}{c}{ Settings } \\
ORACE-Net Tactical Devices & 4 (raspberry pi 2) OS: Raspbian v8.0 \\
Mobile nodes (coordinators) & 2 (Samsung Galaxy S3-I9300-rooted) \\
Wireless mode & OS: Android 4.2.2 CyanogenMod 10.0 \\
ESSID & Ad hoc \\
Wireless standard & CROW2 \\
Transmission power & IEEE 802.11n/2.412 GHz (Channel 1) \\
Experiment area & 0 dBm \\
CC-node connection & 30 m $\times 150$ m \\
Number of iterations & Ethernet to Internet \\
Experimentation duration & Ad hoc WiFi to ORACE-Net network \\
\hline \multicolumn{1}{c}{ ORACE-Net Protocol and Application Layer Settings } \\
\hline Application layer & 3 \\
MQTT msg size/intervals & MQTT client used for pushing data to the IoT \\
Hello/ADV packet size & platform \\
Hello/ADV intervals & 30 Kb/1 s \\
Multicast address/port & $20 / 25$ Bytes \\
\hline & $3 \mathrm{~s}$ \\
\hline Wireless standard & 224.0.0.0/10000 \\
Shimmer [39] Sensing Device Settings & Bluetooth IEEE 802.15.1 \\
Device/Body & Pressure, Temperature, Gyroscope \\
Buffer [39] & $(x, y, z$, axis-angle), Acceleration $(x, y, z)$, \\
Message interval & Magnetometer $(x, y, z)$, Battery level \\
\hline & 1 (with multiple embedded sensors) \\
& 1024 bytes \\
\hline & $1 \mathrm{~s}$ \\
\hline
\end{tabular}




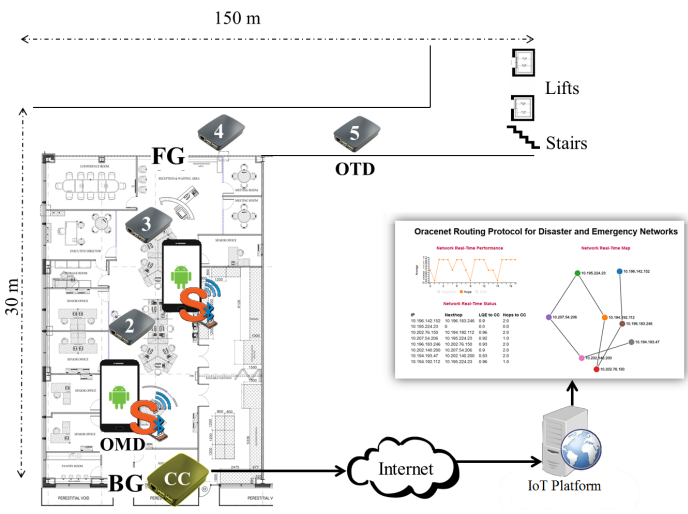

(a)

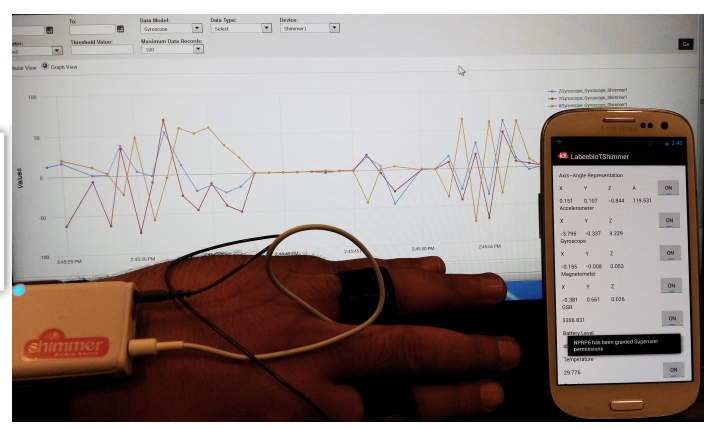

(b)

Figure 5. (a) Map of the experiment scenario; (b) Data collecting from the on-body Shimmer sensors to the android mobile application and then to the IoT platform through the ORACE-Net tactical deployed network. OMD: ORACE-Net mobile device; OTD: ORACE-Net tactical device; FG: front gate of the office; BG: back gate.

Data collected from on-body sensors is routed from WBAN node through the other WBAN node and/or tactical deployed nodes. By reaching the CC node, data is pushed through Internet to the IoT platform. On the IoT platform, data is plotted instantly as depicted by the curves shown in Figure 5b. The WBAN node behavior during the experiment is observed as depicted by Figure 6. The end-to-end link quality estimation (i.e., $E 2 E_{L Q E}$ ) is a real-time metric calculated between a mobile node and the $C C$ node. The bottom curve of Figure 6 illustrates the $E 2 E_{L Q E}$ results over the time. There is a strong correlation between $E 2 E_{L Q E}$ and the $H O P_{\text {Count }}$. It is observed that when the mobile node reaches more than 3 hops away from the CC node, and maintains that HOP Count for more than $2 \mathrm{~s}$, the $E 2 E_{L Q E}$ decreases sharply. When the $E 2 E_{L Q E}$ decreases significantly, connection latency increases and leads to mobile node disconnection. This is due to many factors: (1) signal degradation caused by the fact of being out of range (and no closed node can relay the mobile's data); and (2) the unstable links between the nodes are caused by the interference effected by WiFi access points, wireless extenders and devices inside the office. Equally important, indoor obstacles raised major signal attenuation [40]. It is noteworthy that the delay in milli-seconds (ms) depicted in Figure 6 is reset to zero when a mobile node is disconnected (we consider that a delay higher than $1000 \mathrm{~ms}$ is an immediate disconnection). Hence, this leads us to investigate the accuracy of the delay and disconnection times. For that, we have set up a process to ping the distant CC node every millisecond. The resulted average round-trip time delay and the average end-to-end disconnections per hop count are illustrated in blue and red respectively in Figure 7.
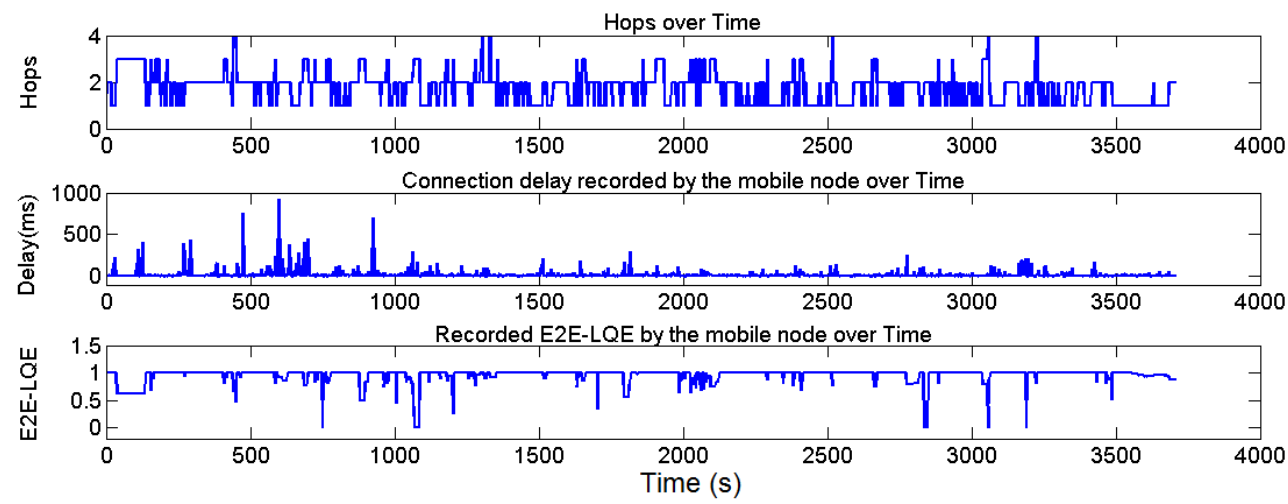

Figure 6. Hop count, instant delay and end-to-end link quality estimation variation during one hour of experimentation for WBAN node in an indoor scenario. 


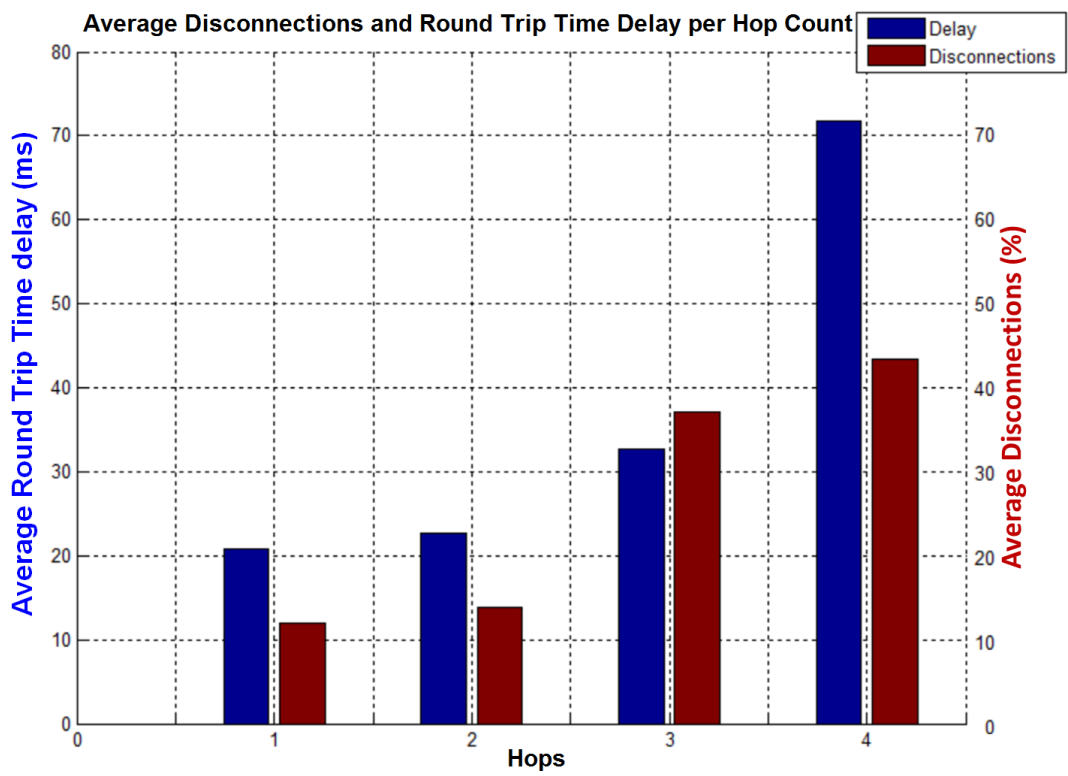

Figure 7. Average disconnections and round-trip time delay per hop count for WBAN (android smart phone mobile node with ORACE-Net protocol-enabled) in an indoor scenario.

What is important to know is that the average percentage of end-to-end disconnections and average round trip time delay increase accordingly with the hop count. With regards to the mobile smartphones used in the experiment (Samsung Galaxy S3 I9300-Battery: 2100 mAh-WiFi IEEE 802.11n), the experimental range is around $100 \mathrm{~m}$. The experiment shows that the best performance is recorded within 1 hop (from the mobile node to the CC node) where average disconnection is around $12 \%$ and average round-trip time delay is equal to $21 \mathrm{~ms}$. However, a connection within 4 hops (approximate distance between two nodes is $45 \mathrm{~m}$ ) makes the average end-to-end disconnections exceed $43 \%$ as illustrated in Figure 7. The average round trip delay increases also to reach $72 \mathrm{~ms}$. It is perceived that for more than 4 hops, average disconnection is expected to exceed $50 \%$.

As has been noted, these results were achieved with intermediate tactical static nodes, hence, results might be worse if all the nodes of the network are mobile. Therefore, based on the best performance recorded (12\% among 1 hop), the standard WiFi IEEE 802.11n remains efficient within short distances only.

Interestingly, we learned from our experiments that the standard WiFi IEEE 802.11n is only appropriate for body-to-body communications in the indoor scenario for an overall distance of 25 to $30 \mathrm{~m}$ (from one node to another). Our results and findings are consistent with the results provided by Andreev et al. in [41]. WiFi IEEE 802.11n remains a candidate for BBN communications for indoor scenarios, but with consideration of the above-cited limitation.

\section{Conclusions and Perspectives}

To conclude, with reference to wearable wireless network end-to-end connectivity, we highlighted the key envisioned challenges. First, while operating at low transmission power and under realistic conditions, the packet reception performance can be significantly degraded while exploiting the BAN-specific IEEE 802.15.6 standard. Second, the routing approach and the dissemination strategy have an impact on the end-to-end connectivity and the end-to-end round-trip time delay. It is concluded also, for an indoor scenario with the considered settings, that the BBN average disconnections are higher than $43 \%$ within 4 hops. Third, it is observed that wireless standard (i.e., WiFi IEEE 802.11n), while having a large coverage support, it is not entirely appropriate for BBN communications in indoor propagation as it has a very high average of end-to-end disconnections.

For the future, it is necessary to investigate other wireless body-to-body communication technologies. Indeed, WiFi IEEE 802.11ac standards, as well as cellular technologies Long-Term 
Evolution (LTE)/5G could potentially fulfill operational BBN requirements (especially regarding range and connectivity). Furthermore, the IEEE 802.11ac standard uses a 5-GHz frequency which avoids most of the interference possibilities and with the provision of higher number of channels defined by the standard (i.e., 25 channels by considering a channel width equal to $20 \mathrm{MHz}$ ) could be interesting to investigate. In addition, 4G/LTE is also another ubiquitous approach followed by $5 \mathrm{G}$ to deliver an edge-free body-to-body experience. Besides, specific technologies targeting the IoT could offer connectivity at various network tiers. LTE Cat M1 Machine Type Communications (eMTC), LTE CAT NB1 Narrowband Internet of Things(NB-IoT), and 802.11ah (WiFi HaLow) are such candidate technologies for which chipsets are known to exist or have been announced. Table 4 highlights a selection of such chipsets. Their availability or future availability paves the way for further investigations and modeling related to their longer range connectivity, reliability, etc. Most of these standards are designed to consume much less power than classical cellular technologies and therefore could prove very effective in future wearable wireless networks.

Table 4. Selected chipsets for upcoming future technologies.

\begin{tabular}{|c|c|c|c|c|c|c|}
\hline Chipset & $\begin{array}{l}\text { Ublox } \\
\text { SARA-N2 } \\
\text { series }\end{array}$ & $\begin{array}{l}\text { Sequans Communication } \\
\text { Monarch SQN3330 }\end{array}$ & $\begin{array}{l}\text { Gemalto } \\
\text { EMS31 }\end{array}$ & $\begin{array}{l}\text { Intel XMM } \\
7115 / \mathrm{XMM} \\
7315\end{array}$ & $\begin{array}{l}\text { Qualcomm } \\
\text { DM9206 }\end{array}$ & $\begin{array}{l}\text { Newarcom } \\
\text { NRC7191 }\end{array}$ \\
\hline Standard(s) & $\begin{array}{l}\text { LTE } \\
\text { Cat NB1 }\end{array}$ & $\begin{array}{l}\text { single-chip LTE } \\
\text { Cat M1/NB1 }\end{array}$ & LTE Cat M1 & $\begin{array}{l}\text { LTE CAT } \\
\text { M1 and NB1 }\end{array}$ & $\begin{array}{l}\text { LTE CAT M1 } \\
\text { and/or NB1 }\end{array}$ & $\begin{array}{l}\text { 802.11ah } \\
\text { (WiFi HaLow) }\end{array}$ \\
\hline Data Rates & $\begin{array}{l}227 \mathrm{kbit} / \mathrm{s} \\
\mathrm{DL} \text { and } \\
21 \mathrm{kbit} / \mathrm{s} \mathrm{UL}\end{array}$ & $\begin{array}{l}\text { up to } 300 \text { kbps DL/375 } \\
\text { kbps UL in HD-FDD and } \\
1 \text { Mbps in FD-FDD (LTE } \\
\text { CAT M1); up to } 40 \text { kbps } \\
\text { DL/55 kbps UL in } \\
\text { HD-FDD (LTE CAT NB1) }\end{array}$ & $\begin{array}{l}\text { up to } 300 \mathrm{kbps} \\
\text { DL and } \\
375 \mathrm{kbps} \text { UL }\end{array}$ & $\mathrm{N} / \mathrm{A}$ & $\mathrm{N} / \mathrm{A}$ & $\begin{array}{l}\text { up to } \\
2 \mathrm{Mbit} / \mathrm{s}\end{array}$ \\
\hline
\end{tabular}

Acknowledgments: This project has received funding from the European Union's Horizon 2020 research and innovation program under grant agreement No 668995. This material reflects only the authors view and the EC Research Executive Agency is not responsible for any use that may be made of the information it contains.

Author Contributions: This paper was prepared through the collective efforts of all authors. In particular, Dhafer Ben Arbia prepared the manuscript based on the suggestions and guidance of Muhammad Mahtab Alam. Dhafer Ben Arbia made substantial contributions towards overall writing, organization, and presentation of the paper. He also conducted the experiment based on which results are discussed. In particular, he contributed in introduction, body-to-body communications: technologies, routing and challenges. He also detailed the experiment scenario and discussed the achieved results. Muhammad Mahtab Alam introduced the scope of the paper within the abstract, then presented the on-body technologies and connectivity challenges with regards to IEEE 802.15.6 standards. Yannick Le Moullec presented the different current implementations of human body communications. He also provided an overview on the future technologies with regards to the IoT trends. Elyes Ben Hamida made contributions to the literature research and paper writing. Muhammad Mahtab Alam, Yannick Le Moullec also did a critical revision of the paper and provided detailed feedback to improve the manuscript content. All authors have read and approved the final manuscript.

Conflicts of Interest: The authors declare no conflict of interest.

\section{References}

1. Alam, M.M.; Ben Hamida, E. Surveying Wearable Human Assistive Technology for Life and Safety Critical Applications: Standards, Challenges and Opportunities. Sensors 2014, 14, 9153-9209.

2. Statistics \& Facts on Wearable Technology. Available online: https://www.statista.com/topics/1556/ wearable-technology / (accessed on 30 June 2017).

3. Sparks, R.; Celler, B.; Okugami, C.; Jayasena, R.; Varnfield, M. Telehealth monitoring of patients in the community. J. Intell. Syst. 2016, 25, 37-53.

4. Perlin, K. Future Reality: How Emerging Technologies Will Change Language Itself. IEEE Comput. Gr. Appl. 2016, 36, 84-89. 
5. Miranda, J.; Cabral, J.; Wagner, S.R.; Fischer Pedersen, C.; Ravelo, B.; Memon, M.; Mathiesen, M. An Open Platform for Seamless Sensor Support in Healthcare for the Internet of Things. Sensors 2016, 16, 2089.

6. Ben Arbia, D.; Alam, M.M.; Attia, R.; Ben Hamida, E. ORACE-Net: A novel multi-hop body-to-body routing protocol for public safety networks. Peer-to-Peer Netw. Appl. 2016, 10, 726-749.

7. Kazemi, R.; Vesilo, R.; Dutkiewicz, E.; Fang, G. Inter-network interference mitigation in Wireless Body Area Networks using power control games. In Proceedings of the 2010 10th International Symposium on Communications and Information Technologies, Tokyo, Japan, 26-29 October 2010; pp. 81-86.

8. Smart Body Area Networks. Available online: http://www.etsi.org/technologies-clusters/technologies/ smart-body-area-networks (accessed on 30 June 2017).

9. Alam, M.M.; Ben Hamida, E. Strategies for Optimal MAC Parameters Tuning in IEEE 802.15.6 Wearable Wireless Sensor Networks. J. Med. Syst. 2015, 39, 1-16.

10. Alam, M.M.; Ben Hamida, E. Wearable Wireless Sensor Networks: Applications, Standards, and Research Trends. In Emerging Communication Technologies Based on Wireless Sensor Networks: Current Research and Future Applications; CRC Press: Boca Raton, FL, USA, 2016; pp. 59-88.

11. Ben Arbia, D.; Alam, M.M.; Attia, R.; Ben Hamida, E. Behavior of wireless body-to-body networks routing strategies for public protection and disaster relief. In Proceedings of the IEEE 11th International Conference on Wireless and Mobile Computing, Networking and Communications (WiMob), Abu Dhabi, UAE, 19-21 October 2015; pp. 117-124.

12. Ben Arbia, D.; Alam, M.M.; Attia, R.; Ben Hamida, E. Implementation and Benchmarking of a Novel Routing Protocol for Tactical Mobile Ad hoc Networks. In Proceedings of the Third IEEE International Workshop on Emergency Networks for Public Protection and Disaster Relief (EN4PPDR 2016), New York, NY, USA, 17-19 October 2016.

13. Chen, M.; Gonzalez, S.; Vasilakos, A.; Cao, H.; Leung, V.C. Body area networks: A survey. Mob. Netw. Appl. 2011, 16, 171-193.

14. Gao, T.; Massey, T.; Selavo, L.; Crawford, D.; Chen, B.R.; Lorincz, K.; Shnayder, V.; Hauenstein, L.; Dabiri, F.; Jeng, J.; et al. The advanced health and disaster aid network: A light-weight wireless medical system for triage. IEEE Trans. Biomed. Circuits Syst. 2007, 1, 203-216.

15. Negra, R.; Jemili, I.; Belghith, A. Wireless Body Area Networks: Applications and Technologies. Proced. Comput. Sci. 2016, 83, 1274-1281.

16. Chen, X.; Xu, Y.; Liu, A. Cross Layer Design for Optimizing Transmission Reliability, Energy Efficiency, and Lifetime in Body Sensor Networks. Sensors 2017, 17, 900.

17. D'Andreagiovanni, F.; Krolikowski, J.; Pulaj, J. A fast hybrid primal heuristic for multiband robust capacitated network design with multiple time periods. Appl. Soft Comput. 2015, 26, 497-507.

18. D'Andreagiovanni, F.; Nardin, A. Towards the fast and robust optimal design of Wireless Body Area Networks. Appl. Soft Comput. 2015, 37, 971-982.

19. Aissi, H.; Bazgan, C.; Vanderpooten, D. Min-max and min-max regret versions of combinatorial optimization problems: A survey. Eur. J. Oper. Res. 2009, 197, 427-438.

20. Maniezzo, V. Exact and approximate nondeterministic tree-search procedures for the quadratic assignment problem. INFORMS J. Comput. 1999, 11, 358-369.

21. Dorigo, M.; Di Caro, G.; Gambardella, L.M. Ant algorithms for discrete optimization. Artif. Life 1999, 5, 137-172.

22. Motwani, R.; Raghavan, P. Randomized Algorithms; Chapman \& Hall/CRC: Boca Raton, FL, USA, 2010.

23. D'Andreagiovanni, F.; Nardin, A.; Natalizio, E. A fast ILP-based Heuristic for the robust design of Body Wireless Sensor Networks. In Applications of Evolutionary Computation; LNCS vol. 10199; Springer: Berlin/Heidelberg, Germany, 2017; pp. 234-250.

24. D'Andreagiovanni, F.; Nace, D.; Nardin, A.; Natalizio, E. Robust relay node placement in body area networks by heuristic min-max regret. In Proceedings of the BalkanCom 2017 Conference, Tirana, Albania, 30 May-2 June 2017.

25. Tsouri, G.R.; Prieto, A.; Argade, N. On increasing network lifetime in body area networks using global routing with energy consumption balancing. Sensors 2012,12, 13088-13108.

26. Pasquero, O.P.; Rosini, R.; D'Errico, R.; Oestges, C. A Correlation Model for Nonstationary Time-Variant On-Body Channels. IEEE Antennas Wirel. Propag. Lett. 2015, 14, 1294-1297. 
27. Mani, F.; D’Errico, R. A Spatially Aware Channel Model for Body-to-Body Communications. IEEE Trans. Antennas Propag. 2016, 64, 3611-3618.

28. Alam, M.M.; Ben Hamida, E.; Ben Arbia, D.; Maman, M.; Mani, F.; Denis, B.; D’Errico, R. Realistic Simulation for Body Area and Body-To-Body Networks. Sensors 2016, 16, 561.

29. Wong, A.; Dawkins, M.; Devita, G.; Kasparidis, N.; Katsiamis, A.; King, O.; Lauria, F.; Schiff, J.; Burdett, A. A 1 V 5 mA multimode IEEE 802.15.6/bluetooth low-energy WBAN transceiver for biotelemetry applications. In Proceedings of the 2012 IEEE International Solid-State Circuits Conference, San Francisco, CA, USA, 19-23 February 2012; pp. 300-302.

30. Wong, A.C.W.; Dawkins, M.; Devita, G.; Kasparidis, N.; Katsiamis, A.; King, O.; Lauria, F.; Schiff, J.; Burdett, A.J. A 1 V 5 mA Multimode IEEE 802.15.6/Bluetooth Low-Energy WBAN Transceiver for Biotelemetry Applications. IEEE J. Solid-State Circuits 2013, 48, 186-198.

31. Zhang, L.; Jiang, H.; Wei, J.; Dong, J.; Li, F.; Li, W.; Gao, J.; Cui, J.; Chi, B.; Zhang, C.; et al. A Reconfigurable Sliding-IF Transceiver for $400 \mathrm{MHz} / 2.4 \mathrm{GHz}$ IEEE 802.15.6/ZigBee WBAN Hubs with Only 21. IEEE J. Solid-State Circuits 2013, 48, 2705-2716.

32. Lee, H.; Lee, K.; Hong, S.; Song, K.; Roh, T.; Bae, J.; Yoo, H.J. A 5.5 mW IEEE-802.15.6 wireless body-area-network standard transceiver for multichannel electro-acupuncture application. In Proceedings of the 2013 IEEE International Solid-State Circuits Conference Digest of Technical Papers, San Francisco, CA, USA, 17-21 February 2013; pp. 452-453.

33. Liu, Y.H.; Huang, X.; Vidojkovic, M.; Ba, A.; Harpe, P.; Dolmans, G.; de Groot, H. A 1.9 nJ/b 2.4 GHz multistandard (Bluetooth Low Energy/Zigbee/IEEE802.15.6) transceiver for personal/body-area networks. In Proceedings of the 2013 IEEE International Solid-State Circuits Conference Digest of Technical Papers, San Francisco, CA, USA, 17-21 February 2013; pp. 446-447.

34. Bachmann, C.; van Schaik, G.J.; Busze, B.; Konijnenburg, M.; Zhang, Y.; Stuyt, J.; Ashouei, M.; Dolmans, G.; Gemmeke, T.; de Groot, H. A 0.74 V $200 \mu \mathrm{W}$ multi-standard transceiver digital baseband in $40 \mathrm{~nm}$ LP-CMOS for 2.4 GHz Bluetooth Smart/ZigBee/IEEE 802.15.6 personal area networks. In Proceedings of the 2014 IEEE International Solid-State Circuits Conference Digest of Technical Papers (ISSCC), San Francisco, CA, USA, 9-13 February 2014; pp. 186-187.

35. Taylor, K. A Modular Transceiver Platform for Human Body Communications. Master's Thesis, Victoria University, Wellington, New Zealand, 2016.

36. Ben Arbia, D.; Alam, M.M.; Attia, R.; Ben Hamida, E. Data Dissemination Strategies for Emerging Wireless Body-to-Body Networks based Internet of Humans. In Proceedings of the Workshop on Advances in Body-Centric Wireless Communications and Networks and Their Applications (BCWNets 2015), Abu Dhabi, UAE, 19 October 2015.

37. Mekikis, P.V.; Kartsakli, E.; Lalos, A.S.; Antonopoulos, A.; Alonso, L.; Verikoukis, C. Connectivity of large-scale WSNs in fading environments under different routing mechanisms. In Proceedings of the 2015 IEEE International Conference on Communications (ICC), London, UK, 8-12 June 2015; pp. 6553-6558.

38. Aschenbruck, N.; Ernst, R.; Gerhards-Padilla, E.; Schwamborn, M. BonnMotion: A Mobility Scenario Generation and Analysis Tool. In Proceedings of the 3rd International Conference on Simulation Tools and Techniques, Malaga, Spain, 16-18 March 2010.

39. Burns, A.; Greene, B.R.; McGrath, M.J.; O’Shea, T.J.; Kuris, B.; Ayer, S.M.; Stroiescu, F.; Cionca, V. SHIMMER: A Wireless Sensor Platform for Noninvasive Biomedical Research. IEEE Sens. J. 2010, 10, 1527-1534.

40. Faria, D.B. Modeling signal attenuation in IEEE 802.11 wireless LANs-Vol. 1. Available online: https: / / pdfs.semanticscholar.org/5d18/474f224f4879a3765598713bae93f9e9c11d.pdf (accessed on 30 June 2017).

41. Andreev, S.; Galinina, O.; Pyattaev, A.; Gerasimenko, M.; Tirronen, T.; Torsner, J.; Sachs, J.; Dohler, M.; Koucheryavy, Y. Understanding the IoT connectivity landscape: A contemporary M2M radio technology roadmap. IEEE Commun. Mag. 2015, 53, 32-40.

(C) 2017 by the authors. Licensee MDPI, Basel, Switzerland. This article is an open access article distributed under the terms and conditions of the Creative Commons Attribution (CC BY) license (http:/ / creativecommons.org/licenses/by/4.0/). 